Review

\title{
Benefits of the Phytoestrogen Resveratrol for Perimenopausal Women
}

\author{
Osamu Wada-Hiraike
}

check for

updates

Citation: Wada-Hiraike, O. Benefits of the Phytoestrogen Resveratrol for Perimenopausal Women. Endocrines 2021, 2, 457-471. https://doi.org/ 10.3390/endocrines2040041

Academic Editor: Antonio Brunetti

Received: 16 August 2021

Accepted: 27 October 2021

Published: 2 November 2021

Publisher's Note: MDPI stays neutral with regard to jurisdictional claims in published maps and institutional affiliations.

Copyright: (C) 2021 by the author. Licensee MDPI, Basel, Switzerland. This article is an open access article distributed under the terms and conditions of the Creative Commons Attribution (CC BY) license (https:// creativecommons.org/licenses/by/ $4.0 /)$.
Department of Obstetrics and Gynecology, Faculty of Medicine, The University of Tokyo, Bunkyo, Tokyo 113-8655, Japan; osamuwh-tky@umin.ac.jp

\begin{abstract}
Endometriosis, characterized by macroscopic lesions in the ovaries, is a serious problem for women who desire conception. Damage to the ovarian cortex is inevitable when lesions are removed via surgery, which finally decreases the ovarian reserve, thereby accelerating the transition to the menopausal state. Soon after cessation of ovarian function, in addition to climacteric symptoms, dyslipidemia and osteopenia are known to occur in women aged $>50$ years. Epidemiologically, there are sex-related differences in the frequencies of dyslipidemia, hypertension, and osteoporosis. Females are more susceptible to these diseases, prevention of which is important for healthy life expectancy. Dyslipidemia and hypertension are associated with the progression of arteriosclerosis, and arteriosclerotic changes in the large and middle blood vessels are one of the main causes of myocardial and cerebral infarctions. Osteoporosis is associated with aberrant fractures in the spine and hip, which may confine the patients to the bed for long durations. Bone resorption is accelerated by activated osteoclasts, and rapid bone remodeling reduces bone mineral density. Resveratrol, a plant-derived molecule that promotes the function and expression of the sirtuin, SIRT1, has been attracting attention, and many reports have shown that resveratrol might exert cardiovascular protective effects. Preclinical reports also indicate that it can prevent bone loss and endometriosis. In this review, I have described the possible protective effects of resveratrol against arteriosclerosis, osteoporosis, and endometriosis because of its wide-ranging functions, including anti-inflammatory and antioxidative stress functions. As ovarian function inevitably declines after 40 years, intake of resveratrol can be beneficial for women with endometriosis aged $<40$ years.
\end{abstract}

Keywords: dyslipidemia; hypertension; osteoporosis; endometriosis; resveratrol; sirtuin

\section{Introduction}

Endometriosis is defined as the growth of the extrauterine endometrial tissue in the presence of estrogen. Although many theories have been proposed to explain its pathophysiology, no definitive conclusion has yet been reached. The frequency of endometriosis is increasing, which is possibly because the frequency of conception in industrialized countries in recent years is lower than that in the early 1990s. In addition, women tend to hesitate to go to hospitals, although symptoms of endometriosis may appear early after menarche. Hence, endometriosis is generally diagnosed later in life, often after years of experiencing symptoms [1]. Unfortunately, the true prevalence of endometriosis in adolescent girls is unknown, and clinical symptoms and diagnostic challenges may arise when young girls are examined. This is mainly due to underestimation of the heavy burden for women who desire conception. Macroscopic lesions of endometriosis most frequently appear in the ovaries. Damage to the ovarian cortex is inevitable while surgically removing the lesions, and ovarian surgery ultimately decreases ovarian reserve, thereby accelerating the menopausal state [2]. Climacteric symptoms, osteopenia, and dyslipidemia occur soon after cessation of ovarian function [3]. Epidemiologically, there are sex-related differences in the frequency of dyslipidemia, hypertension, and osteoporosis. Females are more susceptible to these diseases than males, and their prevention is important for 
extending healthy life expectancy. In premenopausal women, the ovaries are the main source of estrogen. Menstruation is established at puberty, which indicates initiation of the ovulation cycle. Periodic changes in the secretion levels of estrogen, the main source of ovarian granulocyte cells, is observed with ovulation, and secretion of estrogen is generally maintained at high level. However, estrogen production diminishes with age, as the oocyte reserve is exhausted when women reach menopause. The secretion level of the follicle stimulating hormone, a pituitary hormone that promotes ovulation, increases significantly, while estrogen levels decrease significantly (Figure 1).

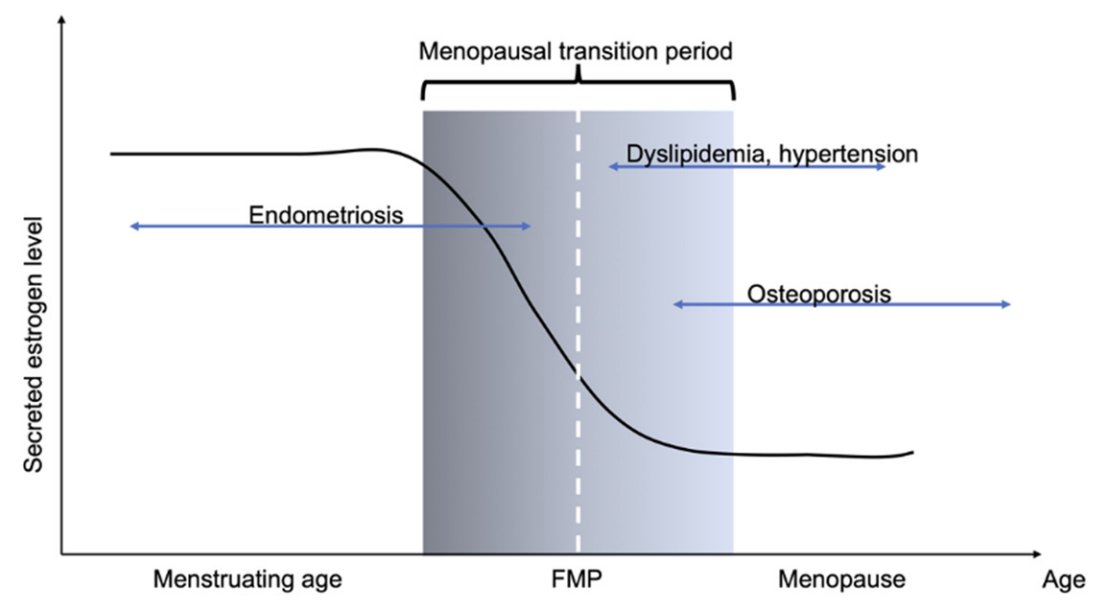

Figure 1. Schematic showing changes in estrogen level with age, and subsequent disease patterns. Menopausal transition period is generally considered between 45 and 55 years. An enigmatic disease, endometriosis is known to occur in women of reproductive age. The burden of endometriosis persists until menopause. Menopausal symptoms and vasomotor symptoms occur soon after cessation of menstruation. This is accompanied by worsening of dyslipidemia and hypertension. Decrease in estrogen level accelerates bone loss because of increase in bone resorption.

Postmenopausal estrogen level is generally low, and its secretion level is maintained by the presence of $\mathrm{P} 450$ aromatase located in peripheral adipose tissues, which converts testosterone into estrogen. Estrogen levels in postmenopausal women are lower than those in men in all generations. Collectively, women experience an inevitable decrease in ovarian function, and the presence of endometriosis accelerates the menopausal stage. It is believed that rapid decrease in estrogen is detrimental for the maintenance of women's health, and that the decrease should be more modest. This theory is known as euestrogenemia [4]. In clinical practice, hormone replacement therapy cannot be prescribed to all women, as it should be optimized individually. Hence, instead using estrogen, safe administration of supplements to all women should be recommended for maintaining a healthy lifestyle.

Dyslipidemia and hypertension are associated with the progression of arteriosclerosis, and arteriosclerotic changes in the large and middle blood vessels are one of the main causes of myocardial and cerebral infarctions. Bone resorption is accelerated by activated osteoclasts, and rapid bone remodeling reduces bone mineral density. Osteoporosis is associated with aberrant fractures in the spine and hip, and the latter confines the patients to the bed. Resveratrol (3,5,40-trihydroxy-trans-stilbene), a plant-derived polyphenol that promotes the function and expression of SIRT1 (discussed later), has been attracting attention, and many reports have shown that resveratrol might exert cardiovascular protective effects. Preclinical reports also indicate that it can prevent bone loss and endometriosis.

In this review, I have discussed the possible protective effects of resveratrol against arteriosclerosis, osteoporosis, and endometriosis because of the wide-ranging functions of resveratrol, including anti-inflammatory and antioxidative stress functions. Thus, as ovarian function inevitably declines in the 40s, resveratrol intake may be beneficial for women with endometriosis in their 40s. 
Sirtuin 1 (SIRT1) is reported to extend the lifespan of yeast and nematodes, and many reports suggest that SIRT1 is a longevity-related gene. The function of SIRT1 is diverse, as it contributes to gene expression control, cell death, stress reaction, aging, and lipid and glucose metabolism. Furthermore, SIRT1 regulates the transcription of estrogen receptors. Activation of SIRT1 function has been studied in animals, as scientists expect it to be involved in life span extension. To achieve the beneficial effect of SIRT1, the use of resveratrol, which is present in red wine, has attracted considerable attention. In this review, we will describe how resveratrol can ameliorate the problems of the cardiovascular system, bone mineral density, and endometriosis, and how these positive effects might be associated with the resveratrol-mediated activation of SIRT1.

\section{Atherosclerosis in Women}

\subsection{Pathophysiology of Atherogenic Event}

Atherosclerosis is macroscopically evident when the artery loses its elasticity due to degenerative changes and becomes hard and thickened. It inevitably occurs due to changes in blood vessels with aging; therefore, it is characterized by thickening of the inner wall of the arterial vasculature [5]. Atherosclerosis is of three types: (1) arteriosclerosis, (2) calcification of mid-sized arteries, and (3) Menkeberg curing. Among them, arteriosclerosis is clinically most significant, as it affects the coronary artery, carotid artery, cerebral artery, and aorta. These large- and medium-sized arteries are directly related to the maintenance of life. In addition, lifestyle-related diseases such as dyslipidemia, hypertension, and diabetes, which are prevalent in Western countries, can adversely affect the progression of atherosclerosis. Prevention of cardiovascular disease is closely related to the prevention of atherosclerosis, as cardiovascular disease is one of the main causes of death in the modern world.

Atherosclerosis develops as follows: at the initial stage, a fat striatum is formed by fat deposition; studies have shown that inflammation in the blood vessels is considerably involved in its formation and development $[5,6]$. Anatomically, the vascular structure is divided into three layers: inner, middle, and outer membranes. In arteriosclerosis foci, a large amount of lipids is deposited between the inner membrane (vascular endothelial) layer and the middle membrane (vascular smooth muscle layer) layer, and tissues containing collagens are observed. Cells leaching into this layer are mainly macrophages that are converted into monocytes after they emerge into the inner membrane. Extracellular matrix-related factors are involved in the interaction between the inner membrane and smooth muscle cells. In addition, low density lipoprotein-cholesterol (LDL-C), which leaches between the inner and middle membrane, is oxidized; macrophages phagocytose the oxidized LDL-C and are transformed into foam cells. During this process, several fat droplets consisting of cholesterol, triacylglycerol, and oxidized LDL-C accumulate in the cytoplasm of macrophages. Foam cells contribute significantly to the deterioration of arteriosclerosis. They deposit lipids in the blood vessels from the remaining foam cells, and oxidized LDL-C directly and adversely affect smooth muscle cells and cytokines, thereby worsening the secretion of vascular endothelial inflammatory cytokines, cell migration, and oxidative stress. Altogether, a strong inflammatory reaction occurs in the vascular endothelial layer. In contrast, the production of nitric oxide (NO), an antioxidant factor, inhibits the secretion of angiogenic factors and inflammatory cytokines, vasoconstriction, production of matrix metalloproteases, which are important for extracellular matrix formation, and the synthesis of adhesive molecules. However, many unsolved questions remain. As atherosclerosis adversely affects the blood vessels of the whole body, the final form of fibrosis, which involves a condition called arterial rupture, is fatal. As described above, arteriosclerosis and stroke can be fatal, because they lead to life-threatening diseases such as myocardial infarction and cerebral infarction. Further elucidation of the conditions and development of novel treatment methods remain important clinical issues. 


\subsection{Sexual Difference and General Therapies of Atherogenesis}

Epidemiologically, the progression of arteriosclerosis differs with gender [7]. Generally, the life expectancy of men is about 10 years lower than that of women. One of the reasons is that men are more likely to die from cardiovascular disease [8]. Furthermore, once cardiovascular disease develops, it is more likely to be more severe in men than in women. However, this changes after menopause $[9,10]$. The blood pressure increases and lipid profile deteriorates, total cholesterol and LDL-cholesterol levels increase, and high density lipoprotein cholesterol levels decrease after menopause. It is presumed that estrogen might have a prophylactic effect on cardiovascular disease, as estrogen secretion significantly decreases after menopause.

The relation between $\mathrm{NO}$ and its production system with estrogen has been clarified at the molecular level. Estrogen receptor (ER) $\alpha$, the main target of estrogen, rapidly directs blood pressure by improving endothelial nitric oxide synthase (eNOS) production. Studies have shown that eNOS expression in pulmonary epithelial cells rapidly rises after estrogen administration [11], which is the result of changes in gene expression after estrogen binds to ERs, and the action of membrane-bound ER $\alpha$ [12]. Furthermore, ER $\alpha$ and ER $\beta$ are expressed in the central nervous system, where they are involved in the expression of neuronal NOS (nNOS). nNOS is also known to contribute to decrease in blood pressure [13]. ER $\alpha$ also controls the expression of kidney-specific angiotensin converting enzyme 2 (ACE2), which inhibits the vasoconstriction effect of angiotensin II (AngII) [14]. The mechanisms of action of estrogen are as follows: (1) regulation of gene expression via ERs, (2) effects of membrane-binding ERs, (3) antioxidant effects of estrogen itself, and (4) effects of ER modification by membrane-bound growth factors. Experiments on model mice and knockout mice have shown that the expression level of ERs in the vascular bed differs with localization [15]. Nonetheless, it is difficult to draw definitive conclusions at present. However, as described later, the phytoestrogen, resveratrol, the mode of action of which resembles that of estrogen, protects the cardiovascular system. Even though the mechanisms of action of estrogen and its receptors are not clearly established, this is an interesting finding.

Although statins are used as standard modes of treatment for lowering LDL-C levels [16], strong statins and proprotein convertase subtilisin/kexin type 9 (PCSK9) did not inhibit the inflammatory response in blood vessels; thus, these agents are currently not used for the complete suppression of cardiovascular events. Inflammation in vascular endothelial cells can be detected using the levels of high-sensitivity C-reactive protein (hsCRP), and it has been suggested that concomitant lowering of LDL-C levels and hsCRP is important for suppressing cardiovascular events [17]. Similar to LDL-C, lowering of the triglyceride level alone is expected to be effective, and several clinical studies using fibrates that mainly lower triglyceride levels have been performed. Large-scale clinical studies such as ACCORD [18] and FIELD [19] did not find any primary preventive effect of fibrates on cardiovascular event development, although the usefulness was shown in subgroup analysis. Other clinical studies using pemafibrate, a peroxisome proliferator-activated receptor $\alpha$ (PPAR $\alpha$ ) agonist, have also been performed [20] and the development of proprietary drugs or ancillary therapeutic agents for suppressing cardiovascular event onset, in addition to conventional treatments such as statins, is awaited. However, in addition to the use of therapeutic agents, it is desirable to monitor and control the progress of arteriosclerosis via daily intake of dietary resveratrol. In this regard, the use of resveratrol in the diet is currently being studied and will be discussed later.

\section{Osteoporosis}

\subsection{Pathophysiology of Osteoporosis}

Bone is histologically divided into cancellous and cortical bone. Cortical bone has dense structure, while cancellous bone consists of thin mesh plates known as trabeculae. The trabeculae are arranged vertically to form a solid structure. Bone mineral density and bone quality are equally important. Bone quality is determined by the characteristics of 
the bone material and its three-dimensional structure. Osteoporosis is characterized by decrease in bone mass and abnormalities in the microstructure of bone tissue, and the overall risk of fracture increases due to increase in bone fragility [21]. Although the bone appears to be a static tissue, it is always formed and resorbed simultaneously, a process called bone remodeling. Abnormalities in bone metabolism occur because of an imbalance between formation and absorption [22]. The old bone is absorbed by osteoclasts, which can construct new bones. Cells, including osteoclasts, osteoblasts, lining cells, and bone cells, should work together for proper bone construction [23]. Osteoclasts differentiate from hematopoietic cells, such as monocytes and macrophages. For osteoclast differentiation, the presence of the NF- $\mathrm{BB}$ activation receptor ligand, RANKL, which is secreted by osteoblasts, is mandatory. RANKL acts by binding to RANK receptors located on the surface of hematopoietic cells. RANKL expression is enhanced by parathyroid hormone, active vitamin D, and inflammatory cytokines, such as interleukin (IL)-1, IL-6, and IL-11. Estrogen performs diverse physiological functions in bone metabolism. It suppresses RANKL expression, thereby suppressing osteoclast differentiation [24]. At the same time, estrogen acts via its receptor, $\mathrm{ER} \alpha$, to exert a wide range of effects, such as suppression of osteocyte and osteoblast death [25]. The canonical Wnt signaling and bone morphogenic protein (BMP) signaling pathways are important for osteoblast differentiation [26]. Osteocytes, differentiated from osteoblasts, suppress bone formation, as they act as antagonists of Wnt signaling by producing sclerostin, a well-known drug discovery target. Osteoblasts that mature and differentiate using the RANKL signal not only adsorb to the bone matrix and decompose inorganic substances from the adsorption surface, but also decompose the proteins of the bone matrix using proteolytic enzymes [24]. The site of completion of bone resorption is called the resorption fossa. After the completion of bone resorption, osteoblasts approach the bone surface of the resorption fossa to produce bone matrix proteins such as type I collagen and osteocalcin, which are located at the same site. The deposition of inorganic mineral components such as calcium and phosphorus results in calcification, which eventually leads to the formation of new bone components in resorption cavities. Several weeks are required for absorption and several months for formation, and the same process is repeated not only during the growth period, but also throughout human life [27]. A balance between bone absorption and formation is necessary for healthy bone metabolism, and the absence of mineral components, which act as the substrates for bone formation, leads to reduction in bone density. The mechanism via which mineral deficiency decreases bone density should be investigated. In addition, the action of excessive PTH promotes bone resorption, resulting in reduction in bone mass.

As bone formation occurs actively in young people $<20$ years of age, the amount of bone obtained during this period is called the peak bone mass (PBM). The epiphyseal cartilage completes bone formation at around the age of 20 years, while bone resorption becomes relatively dominant at around the age of 20-30 years; hence, the overall bone mass is lower than the peak bone mass [28]. However, the extent of the decrease is highest in women during the premenopausal period [29]. PBM should be maximized during the growth stage to prevent osteoporosis, which is especially important for women who are at higher risk of osteoporosis than men. Mechanical pressure and physical activity are the other important factors required for the appropriate formation of bone mass. Disuse or lack of physiological burden on the bone leads to loss of bone mineral density, as is observed even in young patients confined to prolonged bed rest because of injury or immobilization.

\subsection{Sexual Difference and General Therapies of Osteoporosis}

In men, the decrease in bone density is primarily due to reduction in bone formation, as a result of which the trabecular bones thin, although the number of trabecular bones and their interconnected state are relatively maintained. In contrast, in women, estrogen deficiency leads to reduction in cancellous bone; thus, bone resorption increases, which decreases the number of trabecular bones and loss of connectivity between the trabecular bones. Therefore, it is believed that the decrease in bone density in women is significantly 
more detrimental than that in men. Female skeletal development and bone mineral density increase with age, and for the successful acquisition of PBM, secretion of sex steroid hormones (especially estrogen) is essential. As explained above, the resorption phase of bone turnover predominates due to decrease in ovarian function. The effect of menopause on bone quality is important. Collagen, which accounts for approximately $50 \%$ of the bone volume, is known to decrease due to aging and oxidative stress. In addition to the decrease in bone mass, deterioration of bone quality contributes significantly to the establishment of osteoporosis; furthermore, deterioration of the protein quality of the bone matrix and calcification also contributes to the deterioration of bone quality.

The frequency of osteoporosis clearly differs with gender. A previous study estimated the frequency of osteoporosis using the average bone mineral density of young people published by the Japan Society for Bone Metabolism [29]. Estimates showed that 12.8 million people in Japan have osteoporosis. The prevalence of osteoporosis increases with age in both men and women, and women are approximately three times more susceptible to osteoporosis than men. This can be partially explained by the fact that PBM is generally higher in men than in women, and that women show marked bone loss soon after menopause; the sharp decline in bone mineral density in women is because of a drastic decline in estrogen levels. A significant decrease in bone mass during menopause is believed to be associated with significant decrease in the estrogen levels produced by the ovaries. This is not observed in men, as androgen levels in men decline with age, although the rate of decrease is modest.

The pathogenic mechanism of osteoporosis is extremely diverse, and treatment should be considered on the basis of pathological conditions and age. In addition, the presence or absence of existing fractures is important, as fractures are the most preventive treatment outcomes. In addition to drug complications, osteoporosis therapy should be designed considering the background of each patient. Most drugs for treating osteoporosis are bone resorption inhibitors; however, with advancements in medical research and pharmacological development in recent years, a wide variety of drugs can be selected, such as drugs that promote bone formation and those that suppress bone resorption, albeit weakly. At the same time, considering that the lumbar region has cancellous bone and that the proximal part of the femur is a cortical bone, it is necessary to select a drug based on the clinical evidence, as each therapeutic effect differs considerably, and relatively few drugs have been conclusively shown to reduce the risk of proximal femur fractures. Estrogens should be used as baseline therapy for treating osteoporosis in women. As evidence shows that estrogens prevent the onset of osteoporosis, it considerably benefits patients when used appropriately. Administration of active vitamin $\mathrm{D}$ derivatives may be considered in pathological conditions in which calcium balance is negative and bone resorption is enhanced. Bisphosphonates are considered in patients who have long-term accelerated bone resorption and wish to reduce the risk of proximal femur fractures. Denosumab is a neutralizing antibody against RANKL, and the FREEDOM study revealed the potent function of denosumab as a long-term agent [30]. In cases where reduced bone formation is the main cause of osteoporosis, recombinant parathyroid hormone ( $\mathrm{rPTH}$ ) preparations are considered. The rPTH preparation is effective when the bone mineral density in the cancellous bone is significantly reduced, although compliance is required as it involves self-injection. Selective estrogen receptor modulators (SERMs) are suitable for correcting the state of activated bone resorption in the early stage of menopause and can be used for a long period of time, as the extent of suppression of bone turnover is relatively weak. It is noteworthy that estrogens can prevent osteoporosis and osteopenia, whereas similar evidence for other agents is not available. Estrogens can be used as therapeutic agents for ameliorating menopausal symptoms, although long-term administration of estrogens should be used with caution, as estrogens increase the risk of breast cancer, deep vein thrombosis, and coronary heart diseases. In addition, although the risk of breast cancer is reduced if estrogens are started after a certain period after menopause, it may worsen the progression of arteriosclerosis; this observation is known as the timing hypothesis. 
Women who start estrogen therapy soon after cessation of menstruation with less arterial damage may gain cardiovascular protective effects, whereas women with advanced lesions of arteriosclerosis may not gain the beneficial effects of estrogens. Therefore, non-hormonal drugs that can prevent osteoporosis and which can be used easily and safely, are extremely beneficial for the maintenance of women's health. Herein, we show that resveratrol is a candidate for such supplements.

\section{Sirtuin Family Molecules and Resveratrol}

\subsection{Sirtuin Family Molecules and Their Functions}

The silent information regulator 2 gene (Sir2) was first discovered in Saccharomyces cerevisiae as a possible longevity gene, the main function of which was identified to be a nicotinamide adenine dinucleotide (NAD+)-dependent ADP-ribosyltransferases. After the discovery of Sir2, orthologues of sirtuins (silent mating type information regulation 2 homolog) were reported, and seven human sirtuins have been identified (Table 1) [31].

Table 1. Sirtuin family molecules and their properties. Characteristics of human Sirtuins are described. Among them, SIRT1 and SIRT5 are postulated to be primary Sirtuins that respond to resveratrol. (a.a: amino acids, CPS-I: Carbamoyl phosphate synthase I RVT: resveratrol).

\begin{tabular}{|c|c|c|c|c|c|c|}
\hline & Size (a.a) & $\begin{array}{l}\text { Subcellular } \\
\text { Localization }\end{array}$ & $\begin{array}{l}\text { Physiological } \\
\text { Function }\end{array}$ & Target & $\begin{array}{l}\text { Physiological } \\
\text { Functions }\end{array}$ & $\begin{array}{c}\text { Response to } \\
\text { RVT }\end{array}$ \\
\hline SIRT1 & 746 & Nucleus & Deacetylation & $\begin{array}{l}\text { PGC1 } \alpha \\
\text { FOXO1 } \\
\text { NFkB }\end{array}$ & $\begin{array}{c}\text { Metabolism } \\
\text { Anti-inflammation } \\
\text { Anti- } \\
\text { Neurodegeneration }\end{array}$ & $\uparrow$ \\
\hline SIRT2 & 388 & Cytosol & Deacetylation & $\begin{array}{c}\mathrm{H} 4 \\
\alpha \text {-tublin }\end{array}$ & $\begin{array}{c}\text { Cell cycle } \\
\text { Transcription }\end{array}$ & ND \\
\hline SIRT3 & 399 & $\begin{array}{c}\text { Nucleus } \\
\text { Mitochondria }\end{array}$ & Deacetylation & AceCS2 & Metabolism & $\downarrow$ \\
\hline SIRT4 & 314 & Mitochondria & $\begin{array}{l}\text { ADP-ribosyl } \\
\text { Transferase }\end{array}$ & $\mathrm{GDH}$ & Insulin secretion & ND \\
\hline SIRT5 & 310 & Mitochondria & $\begin{array}{l}\text { Desuccinylase } \\
\text { Deacetylation? }\end{array}$ & $\begin{array}{c}\text { Cytochrome C, } \\
\text { CPS-I }\end{array}$ & $\begin{array}{l}\text { Oxidative } \\
\text { metabolism } \\
\text { Apoptosis }\end{array}$ & $\uparrow$ \\
\hline SIRT6 & 355 & Nucleus & $\begin{array}{l}\text { ADP-ribosyl } \\
\text { Transferase }\end{array}$ & $\begin{array}{c}\text { DNA } \\
\text { polymerase } \beta\end{array}$ & DNA repair & ND \\
\hline SIRT7 & 400 & Nucleus & Unknown & $\begin{array}{c}\text { RNA } \\
\text { polymerase I }\end{array}$ & $\begin{array}{c}\text { Transcription } \\
\text { of rDNA }\end{array}$ & ND \\
\hline
\end{tabular}

Phylogenetic analysis of sirtuin family molecules revealed that they can be divided into four subclasses: class I (SIRT1: Ia, SIRT2, and SIRT3: Ib), class II (SIRT4), class III (SIRT5), and class IV (SIRT6: IVa and SIRT7: IVb). They share a common catalytic domain as a unique protein structure and are primarily thought to act as $\mathrm{NAD}^{+}$-dependent histone deacetylases. Sirtuin enzymatic activity results in the formation of deacetylated lysine, nicotinamide, and O-acetyl-ADP-ribose. Among the sirtuin family molecules, SIRT1, SIRT3, and SIRT6 have been proposed to be involved in cardiovascular systems [32]. SIRT1 is primarily an $\mathrm{NAD}^{+}$-dependent deacetylase localized in the nucleus, and recent studies have indicated that SIRT1 can influence intracellular signaling by deacetylating factors such as p53, FOXO, PGC- $1 \alpha, \mathrm{NF}-\mathrm{kB}$, and $\beta$-catenin, thereby regulating gene expression, cell death, stress response, aging, and lipid and glucose metabolism (Figure 2) [33]. 


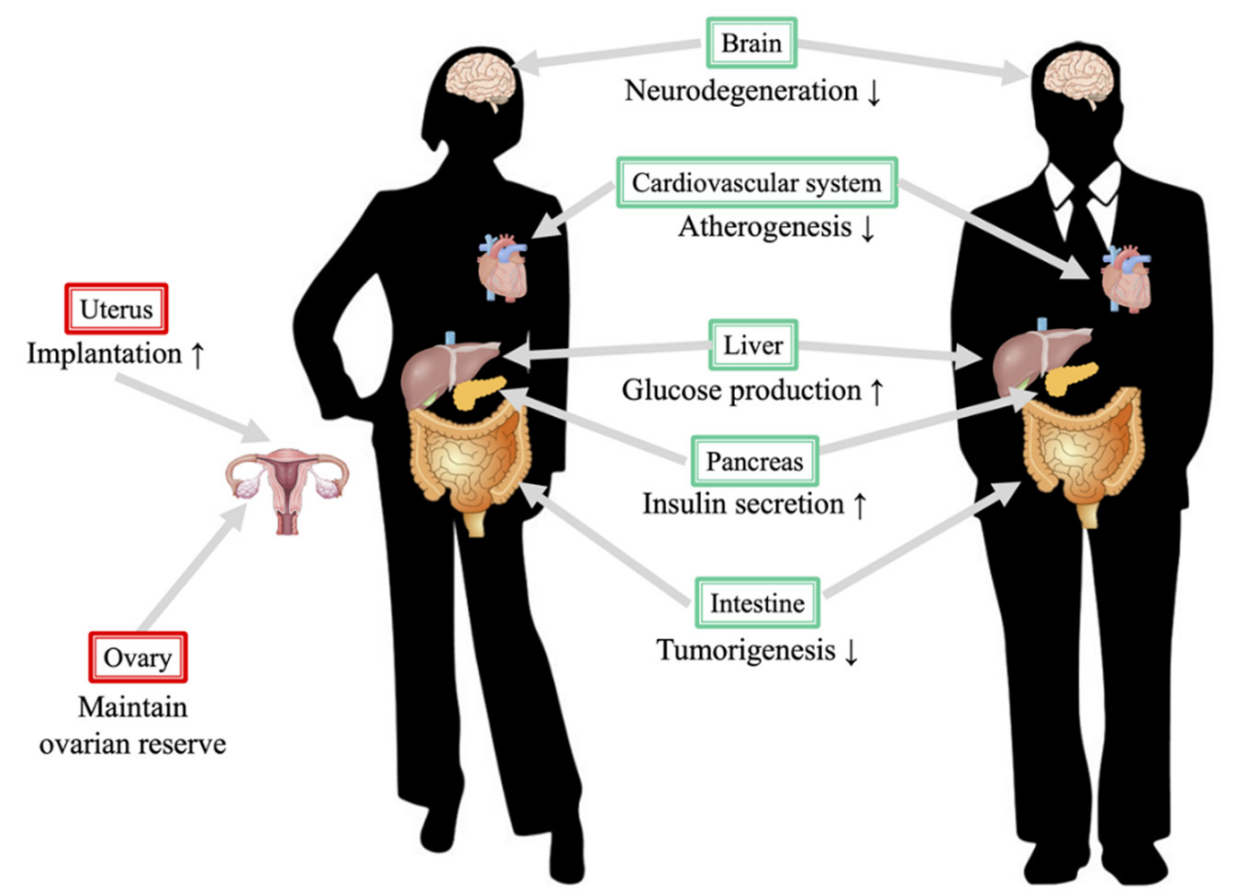

Figure 2. Protective roles of SIRT1 in the human body. The figure on the left denotes a female and that on the right denotes a male. Notably, several functions of SIRT1 in reproductive organs are beneficial. Resveratrol, a SIRT1 activator, promotes the luteinization function in the ovary [34], and several studies indicate that calorie restriction improves ovarian reserve [35]. Another beneficial mechanism is that SIRT1 might improve implantation in females via its effects on adhesion molecules [36].

SIRT1 also regulates autophagy flux by controlling FOXO1 and Rab7, which exerts a protective effect on cardiomyocytes [37]. Cardiac-specific overexpression of SIRT1 in a mouse model revealed that SIRT1 protects cardiac and renal functions affected by endoplasmic reticulum stress [38]. The regulatory mechanism of SIRT1 is complicated, as SIRT1 expression is upregulated during pressure overload. Calorie intake limitation, known as caloric restriction (CR), results in SIRT1 activation, and CR, exercise, and acute ischemic preconditioning stimulate SIRT1 expression. However, many hypotheses have been put forward to explain the mechanism underlying CR and SIRT1 activation; for example, CR promotes $\mathrm{NAD}^{+}$production in cells, which suppresses the production of nicotinamide and promotes the expression of nicotinamide phosphoribosyltransferase and leads to adenosine monophosphate-activated protein kinase (AMPK) activation. CR is known to exert a protective effect against ischemic heart diseases. SIRT1 is known for its pleiotropic function, and we have demonstrated that SIRT1 can control the immune response of endometrial stromal cells by inhibiting TNF $\alpha$-induced IL-6 secretion [39]. Taken together, SIRT1 appears to suppress the progression of endometriotic lesions.

In the sirtuin family, SIRT3 is primarily responsible for antioxidative stress effects and is known to exert a systemic effect, along with SIRT1. SIRT3 knockout mice exhibit systemic aging phenomena, such as deafness, myocardial hypertrophy, and blood vessel fibrosis [40]. Furthermore, it reportedly causes capillary abnormalities in the heart due to mitochondrial dysfunction. Enhancement of SIRT3 expression in cardiomyocytes possibly reduces the risk of heart failure, as it increases the expression of antioxidant factors, reverses fibrosis, and improves vascular endothelial cell function, thereby improving heart function [41]. Many studies have demonstrated the positive effects of SIRT3 on the cardiovascular system. In addition to its cardioprotective effect, recent studies have implicated the possible role of SIRT3 in maintaining bone metabolism, especially in bone resorption. Sirt3 deletion did not affect the skeleton of young mice; however, SIRT3 inhibited osteoclast differentiation, and deletion of SIRT3 increased trabecular bone mass in female mice. Another study also suggested the involvement of SIRT3 in maintaining healthy bone metabolism, as SIRT3 
protects against advanced glycation end product-induced bone marrow mesenchymal stem cell (BMSC) senescence and contributes to improvement of bone mineral density. We speculated that SIRT3 activation might play dual roles in protecting bone metabolism, which varies with the age of the subjects. Although no report has suggested the role of SIRT3 in endometriosis, we have previously shown the role of SIRT3 in the ovaries. Significant oxidative stress appears after SIRT3 depletion in ovarian granulosa cells, which reduced the mRNA levels of aromatase, StAR, 17 $\beta-H S D, p 450 s c c$, and 3 $\beta-H S D$ [42].

As discussed, the sirtuin family has been found to be responsible for specific functions, which depend on the subtype. However, the mechanism of sirtuin stimulation by small-molecule compounds is poorly understood. A previous report using substrates and fluorophore-labeled peptides revealed a possible mechanism via which resveratrol inhibits human SIRT3. This was further confirmed using crystal structure analysis. The compound that activated SIRT3 acted as the top cover, and the binding pocket of the polypeptide was closed as a result of this binding, which enabled efficient and direct interaction with this substrate [43].

\subsection{Function and Efficacy of Resveratrol in Cardiovascular Diseases and Osteoporosis}

Resveratrol, a polyphenol present in red wine, can regulate vascular endothelial cells, pathological growth of smooth muscle cells, infiltration into the vascular walls of immune cells, and vascular remodeling [39,40]. Resveratrol might improve arterial stiffness and lower blood pressure in vivo. Resveratrol suppresses the expression of anti-apoptotic factors by increasing SIRT1 activation and expression both in vivo and in vitro. Resveratrolinduced SIRT1 activation not only increases the expression level of SIRT1 directly, but also enhances intracellular $\mathrm{NAD}^{+}$concentration [44]. In addition, the activation of SIRT1 by resveratrol is also important for controlling autophagy, as autophagy flux positively acts on myocardial protection. In experiments using vascular smooth muscle cells (VSMCs), expression of the AT1 receptor is suppressed by the activation of SIRT1, which is induced by overexpression or resveratrol [45]. Resveratrol also suppresses cell death in cardiomyocytes via Ang II signaling, which is reported to increase superoxide dismutase (SOD) and SIRT1 expression levels [46]. In experiments using 24-month-old aging mice, AngII, prorenin receptor (PRR), and angiotensin converting enzyme (ACE) expression was suppressed by resveratrol administration [47]. Interestingly, the precise molecular mechanism via which resveratrol activates SIRT1 remains controversial [48]. High-throughput screening of chemicals that activate sirtuin revealed more than 14,000 compounds. SRT1720, SRT1460, SRT2183, and resveratrol were used in biochemical assays such as nuclear magnetic resonance, surface plasmon resonance, and isothermal calorimetry, in which they did not show any apparent activation with native peptide or full-length protein substrates [49]. Thus, the mechanism via which resveratrol activates SIRT1 is complicated, and the functions of resveratrol are now considered pleiotropic as they affect multiple receptors and substrates. A computational and biochemical analysis demonstrated that resveratrol can activate the deacetylase activity of SIRT3 and SIRT5, whereas resveratrol can inhibit the desuccinylase activity of SIRT5 [43]. This suggested that resveratrol can modulate sirtuins; thus, determining whether the effects of resveratrol are a central or peripheral issue in the human body is challenging.

In addition, resveratrol activates the transcription of ER as a phytoestrogen. Phytoestrogens can activate ER in the order of approximately $10^{-6} \sim 10^{-5} \mathrm{M}$ in in vitro studies. Generally, phytoestrogens mainly act as ER $\alpha$ antagonist, but can weakly activate ER $\beta$; therefore, phytoestrogens are considered to be SERM [50]. Resveratrol is known to possess polyphenolic properties; similar to catechins, resveratrol might possess antioxidative stress function and may activate nuclear factor-erythroid-derived-derived 2-related factor-2 (Nrf2), which is known to promote potent antioxidative stress effects. In a study using rat VSMCs, resveratrol promoted the expression of SIRT1 and Nrf2, resulting in the suppression of vascular calcification [15]. Nrf2 itself can be activated by resveratrol, and approximately $1 \mathrm{nM}$ resveratrol is required for SIRT1 activation, indicating that the antioxi- 
dant effect of resveratrol depends on Nrf2. Thus, Nrf2 activation by dietary compounds is more realistic than SIRT1 activation, which requires micromolar levels of resveratrol. Nrf2 is a transcriptional factor that is degraded by Keap 1 in the steady state. After exposure to oxidative stress, it translocates to the nucleus and binds to a specific lesion known as the antioxidant responsive element (ARE). After Nrf2 binds to ARE, downstream genes such as $S O D$, glutathione peroxidase (GPX), and heme oxygenase-1 (HO-1) are transcribed, resulting in a clear protective effect on blood vessels. Resveratrol activates SIRT1, ER $\beta$, and Nrf2 concomitantly, and promotes the differentiation of many cell types; thus, resveratrol might have the potential to inhibit cell growth. A recent study indicated that resveratrol activates SIRT1 and SIRT5, while it has been suggested to suppress the function of SIRT3 using computational structural analysis. Resveratrol also contributes to the activation of AMPK [51]. Activation of AMPK has been shown to increase NO production [52] resulting in the activation of eNOS in murine models of high blood pressure [53]. In addition, PPAR $\alpha$ is known to possess anti-inflammatory, anti-angiogenesis, and antioxidative stress properties. Resveratrol is known to activate $\operatorname{PPAR} \alpha$, which results in activation of the AMPK pathway [54]. In a study using human umbilical vein endothelial cells (HUVECs), resveratrol suppressed HUVEC migration and monocyte chemotaxis by inhibiting monocyte chemoattractant protein-1 [55]. Resveratrol not only increased the expression of eNOS via AMPK, but also promoted the phosphorylation of eNOS.

Resveratrol has also been shown to exert beneficial effects on cultured cells, primary mesenchymal stem cells, preosteoblasts, and osteoclast progenitors in in vitro assays. Detailed reviews concluded that resveratrol might perform dual positive roles in bone, as it promotes osteoblastogenesis and inhibits osteoclastogenesis. Considering its dual positive role, treatment with resveratrol is ideal for maintaining bone mineral density in women at risk of osteoporosis. Previous studies have proposed various molecular mechanisms of resveratrol action. Cultured human BMSCs showed increased thymidine incorporation, maturation (as determined using alkaline phosphatase activity assay), and expression of osteoblastic markers (RUNX2/CBFA1, osterix, and osteocalcin). The effects were thought to be similar to those of $17 \beta$ estradiol and were antagonized by ICI182,780, an ER antagonist. Resveratrol induced the activation of AMPK and suppressed the formation of tartrate-resistant acid phosphatase (TRAP)-positive multinucleated cells in mouse bone marrow macrophage-derived osteoclasts. This mechanism was translated as negative regulation of RANKL by AMPK, and its function is related to $\mathrm{Ca}^{2+} /$ calmodulin kinase (CaMK) and TGF- $\beta$-activated kinase-1 (TAK1). Effect of resveratrol on the Wnt signaling pathway has been investigated and resveratrol treatment of BMSCs dose-dependently enhanced $\beta$-catenin nuclear accumulation and positively regulated downstream TCF/LEF transcription, which induced osteoblastic cell differentiation; the increase in $\beta$-catenin accumulation was partially because resveratrol reduced the level of glycogen synthase kinase $3 \beta$. As explained above, resveratrol activated SIRT1 potently, which might play a prominent role in alleviating bone metabolism. One of the underlying mechanisms is that resveratrol promotes the interaction between SIRT1 and p300, a transcription coactivator, and represses RANK expression [56].

Low bioavailability is one of the major problems associated with resveratrol use. Although the concentration of resveratrol that can effectively activate SIRT1 in the experimental system is approximately in the micromolar range, this concentration could not be reached via oral administration. Topical administration may be an alternative approach. Reports show that stenosis of the rat carotid artery improved upon using stents containing resveratrol. If resveratrol is used for treating heart disease, we might expect prevention of arteriosclerosis, cardiac hypertrophy, and myocardial fibrosis. Currently, few reports have shown prevention of blood vessel calcification [15], and further investigation is necessary. As mentioned previously, resveratrol is a powerful natural antioxidant factor with potential for clinical application; in addition, other antioxidant substances might positively affect lipid metabolism. In future, we intend to use resveratrol to suppress cardiovascular events. Many reports have shown that resveratrol is a phytoestrogen; however, other phytoestro- 
gens might also possess cardiovascular protective functions. Plants contain estrogen, such as isoflavones, stilbenes, flavones, and lignans. Among isoflavones, genistein, daidzein, and equol are used to alleviate symptoms of menopause. It is generally known that phytoestrogens can bind to ER $\alpha$ and ER $\beta$ and exhibit both agonistic and antagonistic actions against ER, although their binding capacity to ER $\beta$ is generally stronger than that to ER $\alpha$. Therefore, phytoestrogens are considered to resemble SERM. Phytoestrogens regulate the proliferation of vascular endothelial cells, vascular permeability, NO production, and eNOS expression. It also suppresses the growth of VSMCs and inhibits AngII production.

\subsection{Function and Efficacy of Resveratrol in Endometriosis}

Resveratrol might improve the pathogenesis of endometriosis, and various mechanisms have been proposed using data derived from experimental preclinical studies. We have previously shown that SIRT1 can control the immune response of endometriotic stromal cells by inhibiting TNF $\alpha$-induced IL-8 secretion [39], and that resveratrol can promote TNF-related apoptosis-inducing ligand-induced apoptosis in endometriotic stromal cells [57]. Studies using endometrial cancer cells also showed that resveratrol induced apoptosis but decreased cellular proliferation. Inhibition of COX-2 expression [58] and activation of AMPK pathway [59] are considered as possible mechanisms of action of resveratrol. Women with endometriosis possess poorer antioxidative stress ability, as evidenced by the decreased level of antioxidants in their peritoneal fluid compared to healthy women [60]. An experimental rodent model of endometriosis showed that resveratrol might play crucial roles in the resolution of endometriosis accompanied by improved antioxidative stress ability. Therefore, resveratrol is considered to prevent, treat, and cure endometriosis by attenuating reactive oxygen species. In addition, suppression of inflammatory cytokine secretion and modulation of M1/M2 macrophage polarization [61] may be another possible mechanism that explains the beneficial effects of resveratrol because increased inflammation is thought to induce oxidative stress in the peritoneal cavity [60]. Peritoneal invasion of endometrial tissues is suggested to play a crucial role in the inhibition of cellular invasion. Matrix metalloproteinases (MMPs) and their inhibitors known as tissue inhibitors of metalloproteinases (TIMPs) play fundamental roles in the establishment of peritoneal invasion of endometrial tissues and the ratio of MMPs/TIMPs is suggested to be important to determine the degree of peritoneal invasion [62]. Resveratrol may suppress the expression of MMP-2 and MMP-9 by enhancing SIRT1 expression, thus suppressing the invasion of endometrial tissues [63]. Sufficient blood supply is crucial for the invasion of endometrial tissues; thus, angiogenesis is regarded as an important factor to establish the implantation of ectopic endometrial tissues. Resveratrol could repress the angiogenic process by inhibiting both proangiogenic and angiogenic factors, which includes vascular endothelial growth factor and hypoxia-inducible factor $1 \alpha$. Resveratrol could also inhibit capillary endothelial cell growth, thereby inhibiting new blood vessel formation [64].

Unfortunately, these observations are limited to in vitro or animal studies. Two clinical studies have utilized resveratrol as an adjunctive therapy for endometriosis. A study including 42 surgically confirmed patients with endometriosis prospectively received oral contraceptives (OCs) alone or together with resveratrol at the dose of $30 \mathrm{mg} /$ body [65]. In another randomized clinical trial including 44 surgically confirmed patients with endometriosis, OCs with $40 \mathrm{mg} /$ body resveratrol did not show any additive effect on dysmenorrhearelated pain relief [66]. The former research included women aged 22-37 years, and the treatment period of resveratrol was 2 months, while the latter included women aged 20-50 years, and the treatment period of resveratrol was 42 days. However, the study period for investigating the efficacy of resveratrol might be too short, and these studies used OCs and resveratrol concomitantly. Therefore, the beneficial role of resveratrol may still be ambiguous. Furthermore, these studies included patients with surgically confirmed endometriosis but the clinical details about the presence of endometrioma, deep infiltrating endometriosis, and superficial peritoneal lesions were not illustrated. We opted to use OCs for the treatment of endometriosis or primary dysmenorrhea in women aged less 
than 40 years. In fact, although it is not contraindicated, the World Health Organization recommends the use of OCs as medical eligibility category II for women aged more than 40 years [67]. Considering healthy aging, resveratrol may be used as an adjunctive therapy for maintaining cardiovascular systems and bone metabolism, and for preventing the development of endometriosis.

\section{Conclusions}

Cardiovascular disease is associated with lifestyle-related diseases such as lipid metabolism abnormalities, high blood pressure, and diabetes; thus, cardiovascular disease is associated with aging. Although therapeutic intervention is important for achieving life span extension, arteriosclerosis can be improved by introducing changes in the diet. Osteoporosis is prevalent and inevitable in women with low ovarian reserves. It increases the risk of being bedridden, although early detection is extremely difficult unless bone mineral density is evaluated. For extending the healthy life expectancy of women, it is desirable to initiate convenient and low-risk treatment at young age. The use of resveratrol is presumed to benefit perimenopausal women in three ways as discussed above. For this purpose, activation of SIRT1 using resveratrol might have therapeutic significance. Considering that resveratrol (1) has estrogen-like action, (2) activates sirtuin family of molecules, and (3) possesses potent antioxidant function, it may be considered as a prophylactic agent for reducing medical expenses and extending life span (Figure 3).

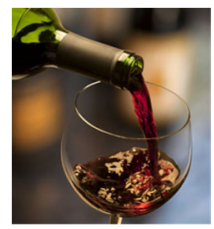

\section{Resveratrol}
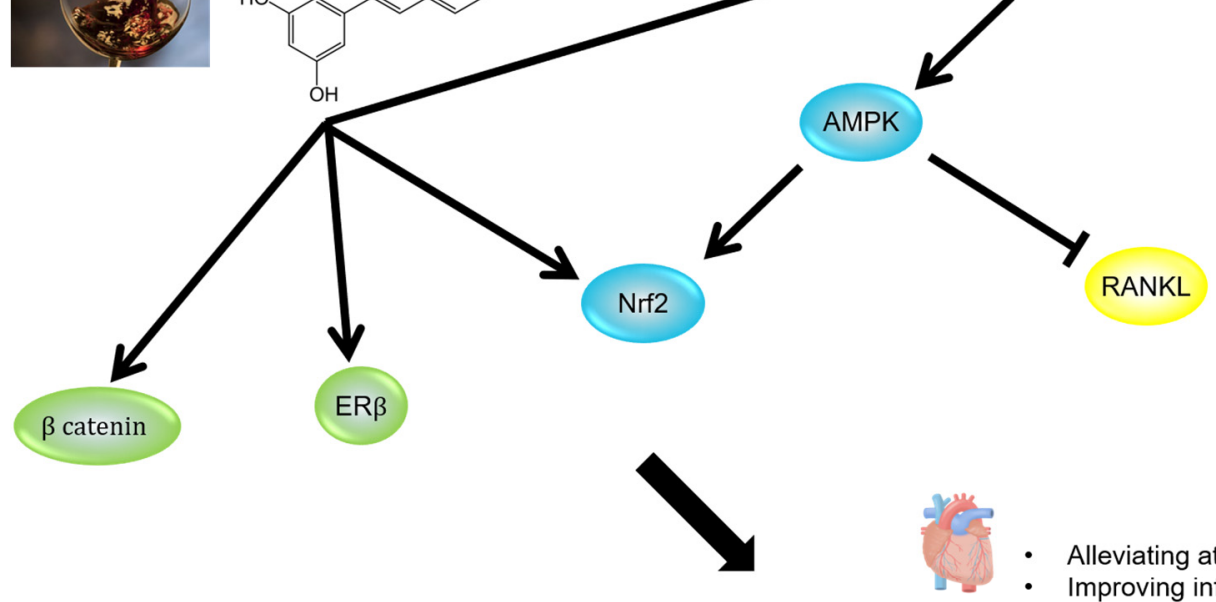

Alleviating atherogenesis

- Improving inflammatory responses

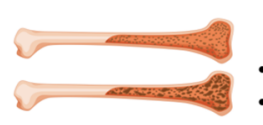

Promotion of osteoblastogenesis Inhibition of osteoclastogenesis

- Inhibition of inflammation

Figure 3. Summary of beneficial functions of resveratrol.

Funding: This work was supported by a Grant-in-Aid for Scientific Research from the Japanese Ministry of Education, Science, and Culture (grant number: 21K09489 (Osamu Wada-Hiraike)) and the Ministry of Health, Labour and Welfare (grant number: 19FB1001 and 20FB1001 (Osamu Wada-Hiraike)).

Conflicts of Interest: The author declares no conflict of interest. 


\section{References}

1. Zondervan, K.T.; Becker, C.M.; Missmer, S.A. Endometriosis. N. Engl. J. Med. 2020, 382, 1244-1256. [CrossRef]

2. Younis, J.S.; Shapso, N.; Ben-Sira, Y.; Nelson, S.M.; Izhaki, I. Endometrioma surgery-A systematic review and meta-analysis of the effect on antral follicle count and anti-Müllerian hormone. Am. J. Obstet. Gynecol. 2021. [CrossRef] [PubMed]

3. Dennerstein, L. A prospective population-based study of menopausal symptoms. Obstet. Gynecol. 2000, 96, 351-358. [CrossRef] [PubMed]

4. Turner, R.J.; Kerber, I.J. A theory of eu-estrogenemia: A unifying concept. Menopause 2017, 24, 1086-1097. [CrossRef] [PubMed]

5. Libby, P.; Ridker, P.M.; Hansson, G.K. Progress and challenges in translating the biology of atherosclerosis. Nature 2011, 473, 317-325. [CrossRef]

6. Geovanini, G.R.; Libby, P. Atherosclerosis and inflammation: Overview and updates. Clin. Sci. 2018, 132, 1243-1252. [CrossRef] [PubMed]

7. Mozaffarian, D.; Benjamin, E.J.; Go, A.S.; Arnett, D.K.; Blaha, M.J.; Cushman, M.; Das, S.R.; de Ferranti, S.; Després, J.-P.; Fullerton, H.J.; et al. Heart Disease and Stroke Statistics-2016 Update: A Report from the American Heart Association. Circulation 2016, 133, e38-e360. [CrossRef]

8. Mendelsohn, M.E.; Karas, R.H.; Yamamoto, Y.; Brady, M.P.; Lu, Z.P.; Maziasz, P.J.; Liu, C.T.; Pint, B.A.; More, K.L.; Meyer, H.M.; et al. Molecular and Cellular Basis of Cardiovascular Gender Differences. Science 2005, 308, 1583-1587. [CrossRef] [PubMed]

9. Tunstall-Pedoe, H. Myth and paradox of coronary risk and the menopause. Lancet 1998, 351, 1425-1427. [CrossRef]

10. Pérez-López, F.R.; Chedraui, P.; Gilbert, J.J.; Pérez-Roncero, G. Cardiovascular risk in menopausal women and prevalent related co-morbid conditions: Facing the post-Women's Health Initiative era. Fertil. Steril. 2009, 92, 1171-1186. [CrossRef] [PubMed]

11. Chen, Z.; Yuhanna, I.S.; Galcheva-Gargova, Z.; Karas, R.H.; Mendelsohn, M.E.; Shaul, P.W. Estrogen receptor $\alpha$ mediates the nongenomic activation of endothelial nitric oxide synthase by estrogen. J. Clin. Investig. 1999, 103, 401-406. [CrossRef]

12. Chambliss, K.L.; Wu, Q.; Oltmann, S.; Konaniah, E.S.; Umetani, M.; Korach, K.S.; Thomas, G.D.; Mineo, C.; Yuhanna, I.S.; Kim, S.H.; et al. Non-nuclear estrogen receptor $\alpha$ signaling promotes cardiovascular protection but not uterine or breast cancer growth in mice. J. Clin. Investig. 2010, 120, 2319-2330. [CrossRef]

13. Xue, B.; Singh, M.; Guo, F.; Hay, M.; Johnson, A.K. Protective actions of estrogen on angiotensin II-induced hypertension: Role of central nitric oxide. Am. J. Physiol. Circ. Physiol. 2009, 297, H1638-H1646. [CrossRef]

14. Brosnihan, K.B.; Hodgin, J.B.; Smithies, O.; Maeda, N.; Gallagher, P.E. Tissue-specific regulation of ACE/ACE2 and AT1/AT2receptor gene expression by oestrogen in apolipoprotein E/oestrogen receptor- $\alpha$ knock-out mice. Exp. Physiol. 2008, 93, 658-664. [CrossRef]

15. Zhang, P.; Li, Y.; Du, Y.; Li, G.; Wang, L.; Zhou, F. Resveratrol Ameliorated Vascular Calcification by Regulating Sirt-1 and Nrf2. Transplant. Proc. 2016, 48, 3378-3386. [CrossRef] [PubMed]

16. Ridker, P.M. Residual inflammatory risk: Addressing the obverse side of the atherosclerosis prevention coin. Eur. Heart J. 2016, 37, 1720-1722. [CrossRef]

17. Ridker, P.M.; Danielson, E.; Fonseca, F.A.; Genest, J.; Gotto, A.M.; Kastelein, J.J.; Koenig, W.; Libby, P.; Lorenzatti, A.J.; MacFadyen, J.G.; et al. Reduction in C-reactive protein and LDL cholesterol and cardiovascular event rates after initiation of rosuvastatin: A prospective study of the JUPITER trial. Lancet 2009, 373, 1175-1182. [CrossRef]

18. Narverud, I.; Ueland, T.; Nenseter, M.S.; Retterstøl, K.; Telle-Hansen, V.H.; Halvorsen, B.; Ose, L.; Aukrust, P.; Holven, K.B. Children with familial hypercholesterolemia are characterized by an inflammatory imbalance between the tumor necrosis factor $\alpha$ system and interleukin-10. Atherosclerosis 2011, 214, 163-168. [CrossRef] [PubMed]

19. D'Emden, M.C.; On behalf of the FIELD Study Investigators; Jenkins, A.J.; Li, L.; Zannino, D.; Mann, K.P.; Best, J.D.; Stuckey, B.G.A.; Park, K.; Saltevo, J.; et al. Favourable effects of fenofibrate on lipids and cardiovascular disease in women with type 2 diabetes: Results from the Fenofibrate Intervention and Event Lowering in Diabetes (FIELD) study. Diabetologia 2014, 57, 2296-2303. [CrossRef]

20. Pradhan, A.D.; Paynter, N.P.; Everett, B.M.; Glynn, R.J.; Amarenco, P.; Elam, M.; Ginsberg, H.; Hiatt, W.R.; Ishibashi, S.; Koenig, W.; et al. Rationale and design of the Pemafibrate to Reduce Cardiovascular Outcomes by Reducing Triglycerides in Patients with Diabetes (PROMINENT) study. Am. Heart J. 2018, 206, 80-93. [CrossRef] [PubMed]

21. NIH Consensus Development Panel on Osteoporosis Prevention, Diagnosis, and Therapy, March 7-29, 2000: Highlights of the conference. South. Med. J. 2001, 94, 569-573.

22. Neve, A.; Corrado, A.; Cantatore, F.P. Osteoblast physiology in normal and pathological conditions. Cell Tissue Res. 2011, 343, 289-302. [CrossRef]

23. Titorencu, I.; Pruna, V.; Jinga, V.V.; Simionescu, M. Osteoblast ontogeny and implications for bone pathology: An overview. Cell Tissue Res. 2014, 355, 23-33. [CrossRef] [PubMed]

24. Khosla, S.; Atkinson, E.J.; Melton, L.J.; Riggs, B.L. Effects of Age and Estrogen Status on Serum Parathyroid Hormone Levels and Biochemical Markers of Bone Turnover in Women: A Population-Based Study. J. Clin. Endocrinol. Metab. 1997, 82, 1522-1527. [CrossRef]

25. Santen, R.J.; Simpson, E. History of Estrogen: Its Purification, Structure, Synthesis, Biologic Actions, and Clinical Implications. Endocrinology 2019, 160, 605-625. [CrossRef] [PubMed]

26. Amjadi-Moheb, F.; Akhavan-Niaki, H. Wnt signaling pathway in osteoporosis: Epigenetic regulation, interaction with other signaling pathways, and therapeutic promises. J. Cell. Physiol. 2019, 234, 14641-14650. [CrossRef] [PubMed] 
27. Parfitt, A.M. Osteonal and hemi-osteonal remodeling: The spatial and temporal framework for signal traffic in adult human bone. J. Cell. Biochem. 1994, 55, 273-286. [CrossRef]

28. Orito, S.; Kuroda, T.; Onoe, Y.; Sato, Y.; Ohta, H. Age-related distribution of bone and skeletal parameters in 1322 Japanese young women. J. Bone Miner. Metab. 2009, 27, 698-704. [CrossRef]

29. Yoshimura, N.; Muraki, S.; Oka, H.; Mabuchi, A.; En-Yo, Y.; Yoshida, M.; Saika, A.; Yoshida, H.; Suzuki, T.; Yamamoto, S.; et al. Prevalence of knee osteoarthritis, lumbar spondylosis, and osteoporosis in Japanese men and women: The research on osteoarthritis/osteoporosis against disability study. J. Bone Miner. Metab. 2009, 27, 620-628. [CrossRef]

30. Bone, H.G.; Wagman, R.B.; Brandi, M.L.; Brown, J.P.; Chapurlat, R.; Cummings, S.R.; Czerwiński, E.; Fahrleitner-Pammer, A.; Kendler, D.L.; Lippuner, K.; et al. 10 years of denosumab treatment in postmenopausal women with osteoporosis: Results from the phase 3 randomised FREEDOM trial and open-label extension. Lancet Diabetes Endocrinol. 2017, 5, 513-523. [CrossRef]

31. Verdin, E.; Hirschey, M.; Finley, L.W.; Haigis, M.C. Sirtuin regulation of mitochondria: Energy production, apoptosis, and signaling. Trends Biochem. Sci. 2010, 35, 669-675. [CrossRef]

32. Sosnowska, B.; Mazidi, M.; Penson, P.; Gluba-Brzózka, A.; Rysz, J.; Banach, M. The sirtuin family members SIRT1, SIRT3 and SIRT6: Their role in vascular biology and atherogenesis. Atherosclerosis 2017, 265, 275-282. [CrossRef]

33. Finkel, T.; Deng, C.-X.; Mostoslavsky, R. Recent progress in the biology and physiology of sirtuins. Nature 2009, 460, 587-591. [CrossRef]

34. Morita, Y.; Wada-Hiraike, O.; Yano, T.; Shirane, A.; Hirano, M.; Hiraike, H.; Koyama, S.; Oishi, H.; Yoshino, O.; Miyamoto, Y.; et al. Resveratrol promotes expression of SIRT1 and StAR in rat ovarian granulosa cells: An implicative role of SIRT1 in the ovary. Reprod. Biol. Endocrinol. 2012, 10, 14. [CrossRef]

35. Xiang, Y.; Xu, J.; Li, L.; Lin, X.; Chen, X.; Zhang, X.; Fu, Y.; Luo, L. Calorie restriction increases primordial follicle reserve in mature female chemotherapy-treated rats. Gene 2012, 493, 77-82. [CrossRef]

36. Shirane, A.; Wada-Hiraike, O.; Tanikawa, M.; Seiki, T.; Hiraike, H.; Miyamoto, Y.; Sone, K.; Hirano, M.; Oishi, H.; Oda, K.; et al. Regulation of SIRT1 determines initial step of endometrial receptivity by controlling E-cadherin expression. Biochem. Biophys. Res. Commun. 2012, 424, 604-610. [CrossRef] [PubMed]

37. Ao, X.; Zou, L.; Wu, Y. Regulation of autophagy by the Rab GTPase network. Cell Death Differ. 2014, 21, 348-358. [CrossRef] [PubMed]

38. Huang, D.; Yan, M.-L.; Chen, K.-K.; Sun, R.; Dong, Z.-F.; Wu, P.-L.; Li, S.; Zhu, G.-S.; Ma, S.-X.; Pan, Y.-S.; et al. Cardiac-Specific Overexpression of Silent Information Regulator 1 Protects Against Heart and Kidney Deterioration in Cardiorenal Syndrome via Inhibition of Endoplasmic Reticulum Stress. Cell. Physiol. Biochem. 2018, 46, 9-22. [CrossRef] [PubMed]

39. Taguchi, A.; Wada-Hiraike, O.; Kawana, K.; Koga, K.; Yamashita, A.; Shirane, A.; Urata, Y.; Kozuma, S.; Osuga, Y.; Fujii, T. Resveratrol suppresses inflammatory responses in endometrial stromal cells derived from endometriosis: A possible role of the sirtuin 1 pathway. J. Obstet. Gynaecol. Res. 2014, 40, 770-778. [CrossRef] [PubMed]

40. Kim, H.-S.; Patel, K.; Muldoon-Jacobs, K.; Bisht, K.S.; Aykin-Burns, N.; Pennington, J.D.; van der Meer, R.; Nguyen, P.; Savage, J.; Owens, K.M.; et al. SIRT3 Is a Mitochondria-Localized Tumor Suppressor Required for Maintenance of Mitochondrial Integrity and Metabolism during Stress. Cancer Cell 2010, 17, 41-52. [CrossRef]

41. Wu, J.; Zeng, Z.; Zhang, W.; Deng, Z.; Wan, Y.; Zhang, Y.; An, S.; Huang, Q.; Chen, Z. Emerging role of SIRT3 in mitochondrial dysfunction and cardiovascular diseases. Free. Radic. Res. 2019, 53, 139-149. [CrossRef]

42. Fu, H.; Wada-Hiraike, O.; Hirano, M.; Kawamura, Y.; Sakurabashi, A.; Shirane, A.; Morita, Y.; Isono, W.; Oishi, H.; Koga, K.; et al. SIRT3 Positively Regulates the Expression of Folliculogenesis- and Luteinization-Related Genes and Progesterone Secretion by Manipulating Oxidative Stress in Human Luteinized Granulosa Cells. Endocrinology 2014, 155, 3079-3087. [CrossRef] [PubMed]

43. Gertz, M.; Nguyen, G.T.T.; Fischer, F.; Suenkel, B.; Schlicker, C.; Fränzel, B.; Tomaschewski, J.; Aladini, F.; Becker, C.; Wolters, D.; et al. A Molecular Mechanism for Direct Sirtuin Activation by Resveratrol. PLoS ONE 2012, 7, e49761. [CrossRef]

44. Xia, N.; Daiber, A.; Förstermann, U.; Li, H. Antioxidant effects of resveratrol in the cardiovascular system. Br. J. Pharmacol. 2017, 174, 1633-1646. [CrossRef]

45. Miyazaki, R.; Ichiki, T.; Hashimoto, T.; Inanaga, K.; Imayama, I.; Sadoshima, J.; Sunagawa, K. SIRT1, a Longevity Gene, Downregulates Angiotensin II Type 1 Receptor Expression in Vascular Smooth Muscle Cells. Arter. Thromb. Vasc. Biol. 2008, 28, 1263-1269. [CrossRef]

46. Tanno, M.; Kuno, A.; Yano, T.; Miura, T.; Hisahara, S.; Ishikawa, S.; Shimamoto, K.; Horio, Y. Induction of Manganese Superoxide Dismutase by Nuclear Translocation and Activation of SIRT1 Promotes Cell Survival in Chronic Heart Failure. J. Biol. Chem. 2010, 285, 8375-8382. [CrossRef] [PubMed]

47. Kim, E.N.; Kim, M.Y.; Lim, J.H.; Kim, Y.; Shin, S.J.; Park, C.W.; Kim, Y.-S.; Chang, Y.S.; Yoon, H.E.; Choi, B.S. The protective effect of resveratrol on vascular aging by modulation of the renin-angiotensin system. Atherosclerosis 2018, 270, 123-131. [CrossRef]

48. Moniot, S.; Weyand, M.; Steegborn, C. Structures, Substrates, and Regulators of Mammalian Sirtuins-Opportunities and Challenges for Drug Development. Front. Pharmacol. 2012, 3, 16. [CrossRef] [PubMed]

49. Pacholec, M.; Bleasdale, J.E.; Chrunyk, B.; Cunningham, D.; Flynn, D.; Garofalo, R.S.; Griffith, D.; Griffor, M.; Loulakis, P.; Pabst, B.; et al. SRT1720, SRT2183, SRT1460, and Resveratrol Are Not Direct Activators of SIRT1. J. Biol. Chem. 2010, 285, 8340-8351. [CrossRef]

50. Bowers, J.L.; Tyulmenkov, V.V.; Jernigan, S.C.; Klinge, C.M. Resveratrol Acts as a Mixed Agonist/Antagonist for Estrogen Receptors $\alpha$ and $\beta$. Endocrinology 2000, 141, 3657-3667. [CrossRef] 
51. Park, S.-J.; Ahmad, F.; Philp, A.; Baar, K.; Williams, T.; Luo, H.; Ke, H.; Rehmann, H.; Taussig, R.; Brown, A.L.; et al. Resveratrol Ameliorates Aging-Related Metabolic Phenotypes by Inhibiting cAMP Phosphodiesterases. Cell 2012, 148, 421-433. [CrossRef]

52. Chen, Z.-P.; Mitchelhill, K.I.; Michell, B.J.; Stapleton, D.; Rodriguez-Crespo, J.I.; Witters, L.A.; Power, D.A.; De Montellano, P.R.O.; Kemp, B.E. AMP-activated protein kinase phosphorylation of endothelial NO synthase. FEBS Lett. 1999, 443, 285-289. [CrossRef]

53. Dolinsky, V.W.; Chakrabarti, S.; Pereira, T.J.; Oka, T.; Levasseur, J.; Beker, D.; Zordoky, B.; Morton, J.S.; Nagendran, J.; Lopaschuk, G.D.; et al. Resveratrol prevents hypertension and cardiac hypertrophy in hypertensive rats and mice. Biochim. Biophys. Acta (BBA)-Mol. Basis Dis. 2013, 1832, 1723-1733. [CrossRef]

54. Okayasu, T.; Tomizawa, A.; Suzuki, K.; Manaka, K.-I.; Hattori, Y. PPAR $\alpha$ activators upregulate eNOS activity and inhibit cytokine-induced NF-kB activation through AMP-activated protein kinase activation. Life Sci. 2008, 82, 884-891. [CrossRef] [PubMed]

55. Cicha, I.; Regler, M.; Urschel, K.; Goppelt-Struebe, M.; Daniel, W.G.; Garlichs, C.D. Resveratrol Inhibits Monocytic Cell Chemotaxis to MCP-1 and Prevents Spontaneous Endothelial Cell Migration Through Rho Kinase-Dependent Mechanism. J. Atheroscler. Thromb. 2011, 18, 1031-1042. [CrossRef]

56. Shakibaei, M.; Buhrmann, C.; Mobasheri, A. Resveratrol-mediated SIRT-1 Interactions with p300 Modulate Receptor Activator of NF- $\mathrm{B}$ Ligand (RANKL) Activation of NF- $\mathrm{B}$ S Signaling and Inhibit Osteoclastogenesis in Bone-derived Cells. J. Biol. Chem. 2011, 286, 11492-11505. [CrossRef]

57. Taguchi, A.; Koga, K.; Kawana, K.; Makabe, T.; Sue, F.; Miyashita, M.; Yoshida, M.; Urata, Y.; Izumi, G.; Tkamura, M.; et al. Resveratrol Enhances Apoptosis in Endometriotic Stromal Cells. Am. J. Reprod. Immunol. 2016, 75, 486-492. [CrossRef] [PubMed]

58. Sexton, E.; Van Themsche, C.; Leblanc, K.; Parent, S.; Lemoine, P.; Asselin, E. Resveratrol interferes with AKT activity and triggers apoptosis in human uterine cancer cells. Mol. Cancer 2006, 5, 45. [CrossRef] [PubMed]

59. Fukuda, T.; Oda, K.; Wada-Hiraike, O.; Sone, K.; Inaba, K.; Ikeda, Y.; Makii, C.; Miyasaka, A.; Kashiyama, T.; Tanikawa, M.; et al. Autophagy inhibition augments resveratrol-induced apoptosis in Ishikawa endometrial cancer cells. Oncol. Lett. 2016, 12, 2560-2566. [CrossRef]

60. Harlev, A.; Gupta, S.; Agarwal, A. Targeting oxidative stress to treat endometriosis. Expert Opin. Ther. Targets 2015, 19, 1447-1464. [CrossRef]

61. Laganà, A.S.; Salmeri, F.M.; Frangez, H.B.; Ghezzi, F.; Vrtačnik-Bokal, E.; Granese, R. Evaluation of M1 and M2 macrophages in ovarian endometriomas from women affected by endometriosis at different stages of the disease. Gynecol. Endocrinol. 2020, 36, 441-444. [CrossRef]

62. Burney, R.O.; Giudice, L.C. Pathogenesis and pathophysiology of endometriosis. Fertil. Steril. 2012, 98, 511-519. [CrossRef] [PubMed]

63. Lee, S.-J.; Kim, M.-M. Resveratrol with antioxidant activity inhibits matrix metalloproteinase via modulation of SIRT1 in human fibrosarcoma cells. Life Sci. 2011, 88, 465-472. [CrossRef]

64. Brâkenhielm, E.; Cao, R.; Cao, Y. Suppression of angiogenesis, tumor growth, and wound healing by resveratrol, a natural compound in red wine and grapes. FASEB J. 2001, 15, 1798-1800. [CrossRef] [PubMed]

65. Maia, H., Jr.; Haddad, C.; Pinheiro, N.; Casoy, J. Advantages of the association of resveratrol with oral contraceptives for management of endometriosis-related pain. Int. J. Women's Health 2012, 4, 543-549. [CrossRef]

66. DA Silva, D.M.; Gross, L.A.; Neto, E.D.P.G.; Lessey, B.A.; Savaris, R.F. The Use of Resveratrol as an Adjuvant Treatment of Pain in Endometriosis: A Randomized Clinical Trial. J. Endocr. Soc. 2017, 1, 359-369. [CrossRef] [PubMed]

67. World Health Organization. Medical Eligibility Criteria for Contraceptive Use Fifth Edition, 2015. Available online: https: / /apps.who.int/iris/bitstream/handle/10665/181468/9789241549158_eng.pdf (accessed on 30 October 2021). 